\title{
ON THE MEASURABLE BOUNDS AND THE DISTRIBUTION OF FUNCTIONAL VALUES OF SUMMABLE FUNCTIONS*
}

\author{
BY
}

\section{CHARLES N. HASKINS}

\section{INTRODUCTION}

1. Object and results. In this paper I define for every function $f(x)$, bounded and summable $\dagger$ on an interval $a \leqq x \leqq b$, or a measurable subset thereof, two numbers, the measurable upper and lower bounds of $f(x)$ on $(a, b)$. These numbers are analogous to the extrema extremorum of a function which is continuous on $(a, b)$, with which in fact they coincide when $f(x)$ is continuous. I show that the measurable bounds can be determined by means of an enumerable set of constants (the " momental corstants ") defined by the Lebesgue integrals

$$
\nu_{n}(f) \equiv \frac{1}{l} \int_{a}^{b}\{f(x)\}^{n} d x
$$

These constants and certain analytic functions determined by them are found to be intimately connected with the structure of the Lebesgue integral of $f(x)$ in the sense that the constants and the analytic functions corresponding to two given functions $f(x)$ and $\phi(x)$ are identical when and only when the defining elements of the Lebesgue integrals of $f(x)$ and $\phi(x)$ are identical. In other words these constants and functions are invariants of all transformations which leave unaltered the defining elements of the Lebesgue integral.

2. The method of Laplace and Darboux. If the functions $f(x)$ and $\phi(x)$ are continuous for $a \leqq x \leqq b$, and $\phi(x)$ assumes its maximum value only once, say at a point $x=c$, it is clear that in general the value of the integral

$$
\int_{a}^{b} f(x)\{\phi(x)\}^{n} d x
$$

is for very large values of $n$ essentially determined by the values of $f(x)$ and $\phi(x)$ in the immediate neighborhood of the point $x=c$. Laplacef used this

* Presented to the Society, October 30, 1915.

†The term "summable" is used as meaning "integrable in the sense of Lebesgue."

‡Laplace, Théorie analytique des probabilités, Cuvres, vol. 7, p. 102. 
principle for the determination of "functions of large numbers." His vaguely stated and, measured by present standards, insufficiently established result was rendered precise, and proved by Darboux* who put it in a form which may be written

$$
\mathbf{L}_{n=\infty} \frac{\sqrt{\frac{n}{\pi}} \int_{a}^{b} f(x)\{\phi(x)\}^{n} d x}{\{\phi(c)\}^{n}}=f(c) \sqrt{\frac{-2 \phi(c)}{\phi^{\prime \prime}(c)}} .
$$

The method is applicable to the case of contour integrals of functions of a complex variable. The case in which $\phi(x)$ assumes its maximum value a finite number of times is reduced to the above mentioned case. Darboux considers only functions $\phi(x)$ having a finite number of discontinuities and those of a simple character.

Stieltjes, $\uparrow$ independently of Darboux, put Laplace's result in essentially the same form but with fewer restrictions on the continuity of $\phi(x)$. He showed by an example that the result may hold when $\phi(x)$ assumes its maximum value an infinite number of times. Lebesgue, $\ddagger$ however, has stated that an example can be constructed in which $\phi(x)$ assumes its maximum value an infinite number of times, and in which the Darboux-Stieltjes result does not hold.

The methods of Darboux have been studied and extended by Flamme, $\$$ Poincaré,|| Féraud, $\uparrow$ Coculesco, ${ }^{* *}$ and Hamy†† with special reference to their application to the problem of determining the approximate values of terms of high order in the development of the perturbative function, and in series relating to the elliptic motion of the planets.

The papers just mentioned (except that of Stieltjes) deal very largely with the case in which $f$ and $\phi$ are complex functions and the integral a contour integral. The singularities of $f$ and $\phi$ are the ordinary isolated singularities of functions which in general are analytic.

3. Singular integrals. The singular integrals which arise in the theory of Fourier's and similar series and the general theory of which has been the

* Darboux, Journal des math ématiques, ser. 3, vol. 4 (1878), p. 32 . Lebesgue, Annales de la Faculté des Sciences de Toulouse, ser. 3, vol..1 (1909), pp. 119-128.

† Stieltjes, Correspondance d'Hermite et de Stieltjes, vol. 2, pp. 185-187.

$\ddagger$ Lebesgue, loc. cit., p. 128.

§ Flamme, Recherche des expressions approchées des termes très éloignés dans les développements du mouvement elliptique des planètes. Thèse (No. 600), Paris, 1887.

|| Poincaré, Les méthodes nouvelles de la mécanique céleste, vol. 1, p. 278, ff.

T Féraud, Sur la valeur approchée des coefficients d'ordre élevé dans les développements en série. Thèse (No. 912), Paris, 1897.

** Coculesco, Journal dees mathématiques, ser. 5, vol. 1 (1895), pp. 359442.

†† Hamy, Ibid., ser. 4, vol. 10 (1894), pp. 39̇1-472; ser. 5, vol. 2 (1896), pp. 381-440. 
object of extensive investigations by Hobson* and Lebesgue $\dagger$ form a broad generalization of the integral of Laplace and Darboux. These investigations relate to integrals of the very general form

$$
\int_{a}^{b} f(t) \phi(t, n, x) d t \quad \text { and } \quad \int_{a}^{b} f(t) \phi(t-x, n) d t,
$$

in which, in some cases at least, $f$ and $\phi$ may have any singularities compatible with integrability in the sense of Lebesgue.

4. Relation of the present paper to those cited in $\S \S 2,3$. The papers above mentioned deal with singular integrals involving two functions $f(t)$ and $\{\phi(t)\}^{n}$ (or $\phi(t, n, x)$ ), and are primarily concerned with the determination of the value of $f(x)$ at a point where $\phi(x)$ (or its absolute value) has a maximum, or with the determination of the functional character of the nucleusfunction $\phi(t, n, x)$ such that

$$
\mathbf{L}_{n=\infty} \int_{a}^{b} f(t) \phi(t, n, x) d t
$$

may represent $f(x)$. In this paper, on the contrary, the singular integrals involve only one function $f(t)$ (or a continuous function of it), and I am concerned not merely with the limiting value of the integral (as $n$ becomes infinite), or its approximation to its limit; but also with the functional character of the integral (or certain functions of it), regarded as depending on the variable $n$. Some of my results are in certain cases (e. g., when $f(x)$ approaches its maximum at only a finite number of points) derivable by specialization of the results of Darboux-Stieltjes. They are not, however, in general so derivable since they hold provided $f(x)$ is merely bounded and summable (or bounded, summable, and positive).

5. Character of functions considered. Throughout the paper the function $f(x)$ will be assumed to have the following properties:

$A$. The function $f(x)$ of the real variable $x$ is defined, real, and single-valued at every point of the interval $a \leqq x \leqq b$, or at every point of a measurable subset $\{x\}$ thereof. (The measure of the domain of definition will be denoted by $l$.)

B. The function $f(x)$ is uniformly bounded on its domain of definition, i. e., there exist two constants $h^{*}$ and $H^{*}$ such that $h^{*} \leqq f(x) \leqq H^{*}$.

$C$. The function $f(x)$ is summable over its domain of definition (and therefore over any measurable subset thereof).

* Hobson, Proceedings of the London Mathematical Society, ser. 2, vol. 6 (1908), pp. 349-395.

†Lebesgue, Annales de la Faculté des Sciences de Toulouse, ser. 3, vol. 1 (1909), pp. 25-117. 


\section{Measurable bounds and momental constants}

6. Measurable bounds of a summable function. Since the function $f(x)$ is summable, the set of points for which $f(x) \geqq y$ is measurable, where $y$ is any real number. Let $M_{y}$ be the measure of this set. Then $0 \leqq M_{y} \leqq l$, and $M_{y}$ is a bounded, monotonic non-increasing function of $y$. The set of points $\{y\}$ for which $h^{*} \leqq y$ and $0<M_{y} \leqq l$ is bounded since $M_{y}=0$ if $y>H^{*}$. Hence this set has a least upper bound $H \leqq H^{*}$. Consequently, however small be the positive quantity $\epsilon$,

$$
M_{H-\epsilon}>0, \quad \text { but } \quad M_{H+\epsilon}=0 \text {. }
$$

Evidently $M_{H}$ may or may not be zero.

The quantity $H$ thus defined I shall call the measurable upper bound of $f(x)$ on its domain of definition.

Similarly we may define the measurable lower bound $h$ of $f(x)$ as a number such that the measure of the set of points for which $f(x) \leqq h+\epsilon$ is positive, but the measure of the set for which $f(x) \leqq h-\epsilon$ is zero, no matter how small $\epsilon$ may be.

The measurable bounds of a bounded summable function always exist. The extension of the definition to unbounded functions is obvious, but of course it does not follow from $H^{*}=\infty$ that $H=\infty$. We may note that, since the alteration of the values of a function at a set of points of measure zero does not change the value of the integral of that function, the measurable bounds of a function are the most general kind of bounds or extremes which we can determine through the instrumentality of a Lebesgue or a Riemann integral.

7. The momental constants, $\nu_{n}(f)$. Since $f(x)$ is bounded and summable, so is also $[f(x)]^{n},(n=0,1,2, \cdots)$, and hence the constants

$$
\nu_{n}(f) \equiv \frac{1}{l} \int_{a}^{b}[f(x)]^{n} d x \quad(n=0,1,2, \cdots),
$$

exist. Evidently $\nu_{0}=1, \nu_{1}=$ integral mean value of $f(x)$. Geometrically $l \nu_{1}, l \nu_{2}, \cdots$ are the several moments of the curve $y=f(x)$ about the axis of $X$, and are therefore analogous to the constants

$$
\mu_{n}(f) \equiv \int_{a}^{b} x^{n} f(x) d x \quad(n=0,1,2, \cdots),
$$

which are the moments of the curve about the axis of $Y$. Since, however, the constants $\nu_{n}(f)$ have a quite different function-theoretic significance from that of the $\mu_{n}(f)$, I propose to call them the momental constants of $f(x)$ on $(a, b)$, as the term moments is already appropriated to the $\mu_{n}(f)$. 
8. The fundamental theorem of the momental constants. ${ }^{*}$ If $f(x)$ and $\phi(x)$ are two real, single-valued, bounded, summable functions defined on the interval $a \leqq x \leqq b$ or measurable subset thereof, the necessary and sufficient condition that their momental constants on that interval (or set) shall satisfy the relations

$$
\nu_{n}(f)=\nu_{n}(\phi) \quad(n=0,1,2, \cdots),
$$

is that the measure of the set of points for which $y_{1}<f(x)<y_{2}$ shall be equal to the measure of the set of points for which $y_{1}<\phi(x)<y_{2}$ for all pairs of real numbers $y_{1}, y_{2}\left(y_{1}<y_{2}\right)$.

The moments $\mu_{n}(f)$ determine the values which a function assumes at the points of its domain of definition (with possible exception of a set of measure zero). $\dagger$ They establish a correspondence between the values $x$ of the domain and the values $y$ of the set of functional values of $f$. In this sense, like Fourier's and other similar constants, they are constants of functional correspondence.

The momental constants $\nu_{n}(f)$, on the other hand, determine the lengths of the sub-intervals (or measure of the subsets) for which $f(x)$ lies between any two prescribed values. They give what may be termed the statistical distribution of the set of functional values of $f(x)$ over its domain of definition and may therefore be called constants of functional distribution. They become constants of functional correspondence when and only when the function $f(x)$ is required to be monotonic non-decreasing (or monotonic nonincreasing). $\ddagger$

9. Schwarz's inequality. In the well-known inequality of Schwarz

$$
\int_{a}^{b}\{\psi(x)\}^{2} d x \int_{a}^{b}\{\chi(x)\}^{2} d x-\left\{\int_{a}^{b} \psi(x) \chi(x) d x\right\}^{2} \geqq 0,
$$

the linear dependence of $\psi(x)$ and $\chi(x)$ save possibly at a set of points of measure zero is an obviously sufficient condition that the equality sign may hold. That it is also a necessary condition has been shown to be true for Riemann integrals,§ and the proof, e. g., by Landsberg's transformation

* The sufficiency of the condition is obvious. The necessity was proved by me for the case of monotonic $f$ and $\phi$ (to which the general case can be reduced) provided one of the functions admits a continuous and positive derivative, which of course is a considerable restriction. The proof was effected by reduction to the theorem of Stieltjes and Lebesgue (An'nales de la Faculté des Sciences de Toulouse, ser. 3, vol. 1 (1909), p. 101) concerning the moments $\mu_{n}(f)$. Prof. Dunham Jackson has, however, proved the theorem in its full generality, and I therefore refer to his note in the present number of these Transactions.

† Lebesgue, loc. cit., p. 102.

‡ See the corollary to the theorem in Prof. Jackson's note.

\$ Landsberg, Math e mat i s che Annalen, vol. 69, 1910, p. 232. Fischer, Archiv für Mathematik und Physik, ser. 3, vol. 13 (1908), pp. 32-40. Richardson and Hurwitz, Bulletin of the American Mathematical S o c i e t y, vol. 16 (1909), p. 18. Curtiss, Ibid., vol. 17 (1911), p. 464. 
$\int_{a}^{b}\{\psi(x)\}^{2} d x \int_{a}^{b}\{\chi(x)\}^{2} d x-\left\{\int_{a}^{b} \psi(x) \chi(x) d x\right\}^{2}$

can be extended to Lebesgue integrals also.*

$$
=\frac{1}{2} \int_{a}^{b} \int_{a}^{b}\left|\begin{array}{l}
\psi(x) \psi(y) \\
\chi(x) \chi(y)
\end{array}\right|^{2} d x d y
$$

10. Application of Schwarz's inequality to the momental constants. Suppose first that $0 \leqq h<H$, that is, that $f(x)$ if negative at all, is so at most at a set of points of measure zero, and further $f(x)$ is not merely a constant (save for a set of points of measure zero).

If in Schwarz's inequality we put

$$
\psi(x)=\{f(x)\}^{(n+1) / 2}, \quad \chi(x)=\{f(x)\}^{(n-1) / 2} \quad(n=0,1,2 \cdots),
$$

we see that

$$
\nu_{n+1}(f) \nu_{n-1}(f)-\left\{\nu_{n}(f)\right\}^{2}>0 \quad(n=0,1,2 \cdots),
$$

and since by the first theorem of the mean

$$
\begin{aligned}
& 0 \leqq h^{n}<\nu_{n}(f)<H^{n}, \\
& \frac{\nu_{n}(f)}{\nu_{n-1}(f)}<\frac{\nu_{n+1}(f)}{\nu_{n}(f)} \quad(n=0,1,2 \cdots) .
\end{aligned}
$$

The inequality sign can be replaced by the equality sign when and only when $h=H$, i. e., when $f(x)$ is constant save for a set of points of measure zero, and the tractions fail to have a meaning when and only when $0=h=H$, i. e., when $f(x)$ has the constant value zero (with possible exception of a set of points of measure zero).

If $h<0$ we may still make the same reduction to Schwarz's inequality provided $n$ be an odd positive integer. In particular we note that

$$
\nu_{2}(f) \nu_{0}(f)-\left\{\nu_{1}(f)\right\}^{2}=\nu_{2}(f)-\left\{\nu_{1}(f)\right\}^{2}>0
$$

in all cases provided $h \neq H$.

III. The distribution functions $R(f, n), P(f, n)$,

$$
J(f, z), K(f, z), L(f, z)
$$

11. The distribution functions $R(f, n), P(f, n)$ for non-negative $f(x)$. In this section we assume $0 \leqq h<H$. Put

Then

$$
R(f, n) \equiv \frac{\nu_{n}(f)}{\nu_{n-1}(f)}, \quad P(f, n)=\left\{\nu_{n}(f)\right\}^{1 / n} \quad(n=1,2,3 \cdots) .
$$

$$
P(f, n)=\{R(f, 1) R(f, 2) \cdots R(f, n)\}^{1 / n} .
$$

* This appears to be a matter of common knowledge, though I do not find specific reference to it in the literature. 
By the first theorem of the mean,

$$
h<R(f, n)<H, \quad h<P(f, n)<H,
$$

and by Schwarz's inequality, ${ }^{*}$

$$
R(f, n)<R(f, n+1) .
$$

Hence by a well-known theorem $\dagger$

$$
P(f, n)<P(f, n+1) .
$$

But since the geometric mean of $n$ different terms lies between the extreme terms

$$
P(f, n)<R(f, n) .
$$

Hence each of the sequences $\{P(f, n)\}$ and $\{R(f, n)\}$ is bounded and monotonic increasing (not merely non-decreasing). Therefore the limits

exist, $\ddagger$ and

$$
\mathbf{L}_{n=+\infty} P(f, n)=H_{1}, \quad \text { and } \quad \mathbf{L}_{n=+\infty} R(f, n)=H_{2}
$$

$$
h<H_{1} \leqq H_{2} \leqq H .
$$

12. The limits of the distribution functions $R(f, n)$ and $P(f, n)$. Let $\epsilon$ be any fixed positive quantity. Let $\left\{x_{\epsilon}\right\}$ be the set of points for which $f(x) \geqq H-\epsilon$ and $\left\{x_{\epsilon}^{\prime}\right\}$ its complementary set. Since $f(x)$ is summable $\left\{x_{\epsilon}\right\}$ and $\left\{x_{\epsilon}^{\prime}\right\}$ are measurable. Let $l_{\epsilon}$ be the measure of $\left\{x_{\epsilon}\right\}$. Then by the definition of $H, l_{\epsilon}$ is positive. Define a function $g_{\epsilon}(x)$ as follows:

Then

$$
\begin{array}{lll}
g_{\epsilon}(x)=H-\epsilon & \text { on } & \left\{x_{\epsilon}\right\}, \\
g_{\epsilon}(x)=0 & \text { on } & \left\{x_{\epsilon}^{\prime}\right\} .
\end{array}
$$

and therefore

$$
0 \leqq g_{\epsilon}(x) \leqq f(x)
$$$$
(a \leqq x \leqq b),
$$

$$
\frac{1}{l} \int_{a}^{b}\left[g_{\epsilon}(x)\right]^{n} d x=\frac{1}{l} \int_{\left\{x_{\epsilon}\right\}}\left[g_{\epsilon}(x)\right]^{n} d x<\frac{1}{l} \int_{a}^{o}[f(x)]^{n} d x=\nu_{n}(f) .
$$

Consequently

$$
\frac{l_{\epsilon}}{l}(H-\epsilon)^{n}<\nu_{n}(f)<H^{n},
$$

and

$$
\left(\frac{l_{\epsilon}}{l}\right)^{1 / n}(H-\epsilon)<P(f, n)<H
$$

* Cf. $\$ 10$.

$\dagger$ Cf. Bromwich, Infinite Series, p. 392, ex. 10 (2).

$\ddagger$ It is of course a consequence of a well-known theorem that if $H_{2}$ exists so does also $H_{1}$, and $H_{2}=H_{1}$. (Cf. Bromwich, Infinite Series, p. 384.) The above form of the proof is chosen in order to show that $H_{1}=H_{2}=H$ and that $R(f, n)$ is a better approximation to the limit than is $P(f, n)$. 
Since $\epsilon$ is fixed, $l_{\epsilon}$ is fixed, and

whence

$$
\mathbf{L}_{n=+\infty}\left(\frac{l_{e}}{l}\right)^{1 / n}=1
$$

$$
H-\epsilon \leqq H_{1}=\mathbf{L}_{n=+\infty} P(f, n) \leqq H_{2}=\mathbf{L}_{n=+\infty} R(f, n) \leqq H
$$

But $\epsilon$ may be taken as small as we please, and consequently

$$
H_{1}=\coprod_{n=+\infty} P(f, n)=\coprod_{n=+\infty} R(f, n)=H_{2}=H .
$$

If $f(x)$ is not bounded on $(a, b)$ it may still happen that $H$ is finite, in which case the foregoing reasoning holds without change. If $H$ is infinite the constants $\nu_{n}(f)$ may not exist if $n>1$. Of course $\nu_{1}(f)$ exists since we suppose $f(x)$ to be summable. If, however, $\nu_{n}(f)$ exists for all positive integral values of $n$, we still have

but

$$
P(f, n)<R(f, n),
$$

$$
\coprod_{n=+\infty} P(f, n)=\coprod_{n=+\infty} R(f, n)=+\infty \text {. }
$$

Suppose now we define the momental constants $\nu_{n}(f)$ for negative integral index $-n$ by the relation

$$
\nu_{-n}(f)=\nu_{n}\left(\frac{1}{f}\right) .
$$

Then if $h>0$ the constants $\nu_{-n}(f)$ surely exist and we have $h<R(f,-n)<R[f,-(n-1)]<P(f,-n)<P[f,-(n-1)]<\frac{1}{\nu_{-1}(f)}$, and finally

$$
h=\mathbf{L}_{n=+\infty} R(f,-n)=\mathbf{L}_{n \doteq+\infty} P(f,-n) .
$$

If $h=0$ the constants $\nu_{-n}(f)$ need not exist for any or for all values of $n$. If they do exist the above relations still hold. Hence we have the following theorem.

13. Theorem. If the lower measurable bound $h$ of a summable function $f(x)$ defined on a finite interval $(a, b)$ or measurable subset thereof is positive, and its upper measurable bound $H$ is finite and different from $h$ then the momental constants

exist. The two functions

$$
\nu_{n}(f) \equiv \frac{1}{l} \int_{a}^{b}\{f(x)\}^{n} d x \quad(n=0, \pm 1, \pm 2, \cdots)
$$

$$
\begin{array}{cc}
P(f, n)=\left[\nu_{n}(f)\right]^{1 / n} & (n= \pm 1, \pm 2, \cdots), \\
R(f, n)=\frac{\nu_{n}(f)}{\nu_{n-1}(f)} & (n=0 \pm 1, \pm 2, \cdots)
\end{array}
$$


are monotonic increasing functions of the integral variable $n$ which satisfy the inequalities

$$
\begin{array}{ll}
h<P(f, n)<R(f, n)<H, & \text { if } n>0, \\
h<R(f, n)<P(f, n)<H, & \text { if } n<0,
\end{array}
$$

and the limiting relations

$$
\begin{aligned}
& \coprod_{n \doteq-\infty} P(f, n)=\coprod_{n \dot{=}-\infty} R(f, n)=h, \\
& \coprod_{n=+\infty} P(f, n)=\coprod_{n \dot{=}+\infty} R(f, n)=H .
\end{aligned}
$$

If $h=0$, or $H=\infty$, or both, the relations are true when the momental constants exist. If $h=H$ the inequalities become equations and the theorem is trivial.

If $h$ and $H$ are of opposite sign the theorem can be applied to find the upper and lower measurable bounds of $[f(x)]^{2}$ and hence of $|f(x)|$.

14. The distribution functions $J(f, z), K(f, z), L(f, z)$ for functions of any sign. The functions $P(f, n), R(f, n)$ of the preceding paragraphs have the disadvantage of requiring $h \geqq 0$. We now proceed to study certain functions analogous to $P$ and $R$ which do not, however, labor under this disadvantage. ${ }^{*}$ In this section we suppose $-\infty<h<H<+\infty$, but make no restriction on the signs of $h$ and $H$.

Let us introduce the auxiliary function of the continuous real or complex variable $z$

Then since

$$
U(f, z)=\frac{1}{l} \int_{a}^{b} e^{z f(x)} d x
$$

$$
e^{z f(x)}=\sum_{n=0}^{n=\infty} \frac{z^{n}\{f(x)\}^{n}}{n !}
$$

the convergence being uniform for all finite $z$ and all $x$ for which $f(x)$ is defined, we have

$$
U(f, z)=\sum_{n=0}^{n=\infty} \frac{\nu_{n}(f) z^{n}}{n !}
$$

and $U(f, z)$ is an analytic, and in fact an integral transcendental function of $z$. It obviously has no real roots. $\dagger U(f, z)$ is completely determined by the momental constants, and, conversely, it completely determines them.

We now define two functions $J(f, z), K(f, z)$ with properties analogous to those of $P(f, n), R(f, n)$ by the relations

* Were it not that the functions $R, P$ depend much more directly on the momental constants $\nu$ than do the functions $J, K, L$, it would hardly be worth while to study them in detail.

$\dagger$ But it may have complex roots. If $a=-1, b=+1, f(x)=x, U(f, z)=(\sinh z) / z$ and has the roots $z= \pm n \pi i(n=1,2,3, \cdots)$. 


$$
\begin{aligned}
& J(f, z)=\frac{1}{z} \ln U(f, z)=\frac{1}{z} \ln \frac{1}{l} \int_{a}^{b} e^{z f(x)} d x \\
& K(f, z)=\frac{1}{U(f, z)} \frac{d}{d z} U(f, z)=\frac{d}{d z} \ln \{U(f, z)\}=\frac{\int_{a}^{b} \frac{e^{z f(x)} f(x) d x}{\int_{a}^{b}} \frac{e^{z f(x)} d x}{}}{}
\end{aligned}
$$

The only singularities of $J(f, z)$ and $K(f, z)$ occur at the (necessarily complex) roots of $U(f, z)$. They are poles of the first order for $K(f, z)$ and logarithmic singularities for $J(f, z)$.

We shall be particularly interested in the behavior of $J(f, z)$ and $K(f, z)$ as $z$ traverses all real values from $-\infty$ to $+\infty$. If however we wish a function having properties analogous to those of $J$ and $K$ but for which $z$ is restricted to the original range $(a, b)$ on which $f(x)$ was defined we may use

$$
L(f, z)=\frac{\int_{a}^{b}\left(\frac{z-a}{b-z}\right)^{f(x) / 2} f(x) d x}{\int_{a}^{b}(\overline{z-a})^{f(x)^{/ 2}} d x}
$$

15. Formulæ relating to $U(f, z), J(f, z), K(f, z)$. In this section I set down certain formulæ involving $U, J$, and $K$, some because they are used in later sections and others because of their intrinsic interest. The proofs are omitted on account of their simplicity.

$$
\begin{gathered}
J(f, z)=\frac{1}{z} \ln U(f, z)=\frac{1}{z} \int_{0}^{z} K(f, z) d z \\
K(f, z)=\frac{d}{d z} \ln U(f, z)=z^{\frac{d J(f, z)}{d z}+J(f, z)} \\
\frac{d}{d z}\left(\frac{z^{n} d^{n-1} J(f, z)}{d z^{n-1}}\right)=z^{n-1} \frac{d^{n-1} K(f, z)}{d z^{n-1}}
\end{gathered}
$$

If $c$ be a constant,

$$
\begin{array}{cc}
K(f+c, z)=K(f, z)+c, & c K(f, c z)=K(c f, z) ; \\
J(f+c, z)=J(f, z)+c, \quad c J(f, c z)=J(c f, z) . \\
K(f, z)=\sum_{n=0}^{n=\infty} \frac{k_{n}(f) z^{n}}{n !}, \quad J(f, z)=\sum_{n=0}^{n=\infty} \frac{k_{n}(f) z^{n}}{(n+1) !}
\end{array}
$$

where 


$$
\begin{aligned}
& k_{0}(f)=\nu_{1}(f) \text {, } \\
& k_{1}(f)=\nu_{0}(f) \nu_{2}(f)-\nu_{1}^{2}(f) \quad\left(\nu_{11}(f)=1\right), \\
& k_{2}(f)=\nu_{0}^{2}(f) \nu_{3}(f)-3 \nu_{0}(f) \nu_{1}(f) \nu_{2}(f)+2 \nu_{1}^{3}(f), \\
& \nu_{n+1}(f)=\sum_{\lambda=0}^{\lambda=n}\left(\begin{array}{c}
n \\
\lambda
\end{array}\right) \nu_{n-\lambda}(f) k_{\lambda}(f) \text {. }
\end{aligned}
$$

16. Monotonic character of $J(f, z)$ and $K(f, z)$ for real $z$. We have

$$
\frac{d}{d z} K(f, z)=\frac{\int_{a}^{b} e^{z f(x)}(f(x))^{2} d x \int_{a}^{b} e^{z f(x)} d x-\left\{\int_{a}^{b} e^{z f(x)} f(x) d x\right\}^{2}}{\left\{\int_{a}^{b} e^{z f(x)} d x\right\}^{2}}
$$

To the numerator we may apply the inequality of Schwarz* with

$$
\psi(x)=e^{(z / 2) f(x)} f(x), \quad \chi(x)=e^{(z / 2) f(x)} .
$$

Hence since $h \neq H$

$$
\frac{d}{d z} K(f, z)>0
$$

and $K(f, z)$ is a monotonic increasing (not merely non-decreasing) function of $z$ for all real $z$.

Now from formula (3) of $\S 15$

Hence

$$
\frac{d}{d z}\left\{z^{2} \frac{d J(f, z)}{d z}\right\}=z \frac{d K(f, z)}{d z} .
$$

But since

$$
\operatorname{sgn}\left\{\frac{d}{d z} z^{2} \frac{d J}{d z}(f, z)\right\}=\operatorname{sgn} z
$$

$$
\begin{aligned}
z^{2} \frac{d J}{d z}(f, z)= & {\left[\frac{\nu_{2}(f)-\nu_{1}^{2}(f)}{2}\right] z^{2}+\cdots, } \\
& z^{2} \frac{d J}{d z}(f, z)>0,
\end{aligned}
$$

except at $z=0$ where it has its minimum value zero. Hence

$$
\frac{d J(f, z)}{d z}>0
$$

except possibly at $z=0$.

* Cf. $\$ 10$. 
But

$$
\left.\frac{d J}{d z}(f, z)\right]_{z=0}=\frac{\nu_{2}(f)-\left(\nu_{1}(f)\right)^{2}}{2},
$$

and this is positive by Schwarz's inequality $(\$ 10)$.

Therefore for all real $z$

$$
\frac{d J(f, z)}{d z}>0
$$

and $J(f, z)$ is a monotonic increasing function of $z$.

Moreover, by (2) of $\S 15$,

and therefore

$$
K(f, z)-J(f, z)=z \frac{d J}{d z}(f, z),
$$

$$
\begin{array}{ll}
K(f, z)>J(f, z) & (z>0), \\
K(f, 0)=J(f, 0)=\nu_{1}, & \\
K(f, z)<J(f, z) & (z<0) .
\end{array}
$$

17. Limits of the distribution functions $J(f, z), K(f, z)$. By the first theorem of the mean

$$
h<J(f, z)<H, \quad h<K(f, z)<H,
$$

for all real $z$. Hence the limits

exist, and

$$
\begin{array}{ll}
\mathbf{L}_{z=-\infty} K(f, z)=h^{\prime \prime}, & \mathbf{L}_{z=+\infty} K(f, z)=H^{\prime \prime}, \\
\mathbf{L}_{\dot{=}-\infty} J(f, z)=h^{\prime}, & \mathbf{L}_{z=+\infty} J(f, z)=H^{\prime},
\end{array}
$$

$$
h \leqq h^{\prime \prime} \leqq h^{\prime}<\nu_{1}<H^{\prime} \leqq H^{\prime \prime} \leqq H .
$$

Now if as in $\S 12$ we let $\left\{x_{e}\right\}$ be the set of points for which $f(x) \geqq H-\epsilon$, and $\left\{x_{e}^{\prime}\right\}$ its complementary set, and if we put

we have for positive $z$

$$
\begin{array}{ll}
g_{e}(x, z)=e^{z(H-\epsilon)} & \left(\text { on }\left\{x_{e}\right\}\right), \\
g_{e}(x, z)=0 & \left(\text { on }\left\{x_{e}^{\prime}\right\}\right) ;
\end{array}
$$

$$
0<g_{\mathrm{e}}(x, z) \leqq e^{z f(x)},
$$

and may show, by reasoning entirely similar to that of $\S 12$, that

$$
H^{\prime}=H^{\prime \prime}=H \text {. }
$$

From $\S 15$, formula 4, we have 


$$
\begin{gathered}
K(f, z)=-K(-f,-z), \\
J(f, z)=-J(-f,-z),
\end{gathered}
$$

from which follows at once that

$$
h^{\prime}=h^{\prime \prime}=h \text {. }
$$

The results thus far obtained may be summarized as follows.

18. Theorem. If on the finite interval $(a, b)$ or measurable subset $\{x\}$ thereof, the function $f(x)$ is vounded and summable, the two functions*

$$
K(f, z)=\frac{\int_{a}^{b} e^{z f(x)} f(x) d x}{\int_{a}^{b} e^{z f(x)} d x}, \quad J(f, z)=\frac{1}{z} \ln \frac{1}{l} \int_{a}^{b} e^{z f(x)} d x
$$

are analytic functions of the complex variable $z$ in the entire finite $z$ plane, except possibly for certain points which are poles of the first order of $K(f, z)$ and logarithmic singularities of $J(f, z)$. For all real finite values of $z, K(f, z)$ and $J(f, z)$ are analytic and monotonic increasing functions of $z$. If $h, \nu_{1}$, and $H$ $(h<H)$, are respectively the lower measurable bound, the integral mean value, and the upper measurable bound of $f(x)$ on its domain of definition, the following relations are true:

$$
\begin{aligned}
& h<K(f, z)<J(f, z)<\nu_{1}<H \quad(z<0) \text {, } \\
& K(f, 0)=J(f, 0)=\nu_{1}, \\
& h<\nu_{1}<J(f, z)<K(f, z)<H \quad(z>0) \text {, } \\
& \mathbf{L}_{z=-\infty} J(f, z)=\coprod_{z \dot{=}-\infty} K(\boldsymbol{f}, z)=h, \\
& \coprod_{z=+\infty} J(f, z)=\mathbf{\coprod}_{z \dot{j}+\infty} K(f, z)=H .
\end{aligned}
$$

If $h=H$ the inequalities become equations and the theorem is trivial.

19. The functions $J(f, z), K(f, z)$ and the distribution of functional values. The functions $J(f, z), K(f, z)$, and $U(f, z)$ completely determine one another, and $U(f, z)$ completely determines and is determined by the momental constants $\nu_{n}(f)$. Hence, we have, making use of the results of $\$ 8$, the theorem stated as follows.

Theorem. Two functions $K(f, z)$ and $K(\phi, z)$ (or $J(f, z)$ and $J(\phi, z)$ ) corresponding to two bounded summable functions $f(x)$ and $\phi(x)$ defined on

* Here $\int_{a}^{b}$ is to be interpreted as the integral over the domain of definition of $f(x)$, be it $(a, b)$ or $\{x\}$, and $l$ is the measure of that domain. 
the same finite interval $(a, b)$ or subset $\{x\}$ thereof are identical when and only when the measure of the set of points for which $g_{1}<f(x)<y_{2}$ is for all pairs of real numbers $y_{1}$ and $y_{2}\left(y_{1}<y_{2}\right)$ equal to the measure of the set for which $y_{1}<\phi(x)<y_{2}$.

It follows that the set of all bounded summable functions defined on $(a, b)$ (or $\{x\}$ ) can be divided into classes each of which is characterized by the enumerable set of momental constants $\nu_{n}$ or by either of the analytic functions $J, K$. Two functions of the set belong to the same class when and only when their Lebesgue integrals have the same infinitesimal structure. The typical representative of a class is a monotonic increasing function which is uniquely determined save for its values at an enumerable set of points.*

* Cf. the corollary in Prof. Jackson's note.

Dartmouth College, Hanover, N. H.,

November 19, 1915 